Pacific Journal of Mathematics

CONVEXITY PROPERTIES OF A GENERALIZED NUMERICAL 


\title{
CONVEXITY PROPERTIES OF A GENERALIZED NUMERICAL RANGE
}

\author{
JOHN DE PILLIS
}

\begin{abstract}
A numerical range $W_{n}(A)$ of a bounded linear operator $A$ on Hilbert space $\mathscr{H}$ is defined to be the set of complex numbers $W_{n}(A)=\{\operatorname{tr}(A M)$ : dimension $M=n\}$ where $M$ runs over all orthogonal $n$-dimensional projections on $\mathscr{H}$, and $\operatorname{tr}(\cdot)$ is the trace functional. It is known that $W_{n}(A)$ is always convex (the Hausdorff-Toeplitz theorem tells us that $W_{1}(A)$ is convex). In what follows, we replace the trace functional by the more general elementary symmetric functions, and derive certain convexity results.
\end{abstract}

The classical Haudorff-Toeplitz theorem has it that for any bounded linear operator $A$ on Hilbert space $\mathscr{H}$, the numerical range

$$
W(A)=\{\langle A x, x\rangle:\|x\|=1\}
$$

is a convex subset of the Complex plane (cf. [4], [9], [10]).

Let $P_{x}$ denote the orthogonal projection $P_{x} ; y \rightarrow\langle y, x\rangle x$ onto the one-dimensional subspace spanned by $x$. Then $\langle A x, x\rangle$ can be shown to equal $\operatorname{tr}\left(A P_{x}\right)$, the trace of the operator $A P_{x}$ (equivalently $\langle A x, x\rangle=$ $\operatorname{tr}\left(P_{x} A P_{x}\right)$, the trace of the compression of $A$ to the space $\mathrm{sp}[x] \mathrm{span}-$ ned by $x$.)

In light of the above interpretation, it is natural to ask whether the set

$$
W_{n}(A)=\{\operatorname{tr}(A M): \text { dimension } M=n\},
$$

where $M$ runs over all $n$-dimensional orthogonal projections on $\mathscr{H}$, is convex; as a convenient ambiguity, we use the symbol $M$ to represent both the $n$-dimensional subspace $M$ and the orthogonal projection on $\mathscr{H}$ whose range is $M$. This question seems to have been raised first by Halmos [5], and consequently answered in the affirmative, by C. A. Berger [1], [6]. The convexity of $W_{n}(A)$ when $A$ is normal was proved by R. C. Thompson [11, Theorem 2], which appeared almost simultaneously with Berger's thesis [1].

In this paper, we extend the notion of $n^{\text {th }}$ order numerical range (1.1) by replacing the linear trace functional $\operatorname{tr}(\cdot)$ by the more general elementary symmetric functions

$$
E_{1}(\cdot)=\operatorname{tr}(\cdot), E_{2}(\cdot), \cdots, E_{r}(\cdot), \cdots, E_{n}(\cdot)=\operatorname{determinant}(\cdot),
$$

defined on the compressions of the operator $A$ to $n$-dimensional sub- 
spaces of $\mathscr{C}$. That is we shall study the set complex of scalars

$$
W_{r, n}(A)=\left\{E_{r}(A M): M \subset \mathscr{H}, \operatorname{dimension}(M)=n\right\} .
$$

First, a quasi-convexity is shown for the set (1.2) for $n=r$ and with $A$ replaced by $A+z$ for large complex $z$ (Theorem 5.2). Then, we present a convexity statement (Theorem 6.1) for those linear operators which exhibit a certain kind of strong convergence (Definition 3.4).

It is somewhat surprising that the badly nonlinear elementary symmetric functions allow for any convexity properties relative to (1.2) at all, but we are able to prove as our principal result, the following property of $W_{r, n}(A)$. (See Theorem 6.2.)

THEOREM. Let $A$ be any bounded linear operator on Hilbert space $\mathscr{H}$ and let $r$ and $n$ be any positive integers such that $1 \leqq r \leqq n$. Then for $E_{r}(A M)$ and $E_{r}(N)$ in $W_{r, n}(A)$, the entire line segment

$$
\left\{\lambda E_{r}(A M)+(1-\lambda) E_{r}(A N): 0<\lambda<1\right\}
$$

is also in $W_{r, n}(A)$ provided the n-dimensional subspaces $M$ and $N$ are mutually orthogonal. More specifically, for every pair of mutually orthogonal $n$-dimensional subspaces, $M$ and $N$, and for every $\lambda \in(0,1)$, there exists an n-dimensional subspace $U_{\lambda}$ such that

$$
\lambda E_{r}(A M)+(1-\lambda) E_{r}(A N)=E_{r}\left(A U_{\lambda}\right) .
$$

Due to the constructive nature of our proof, we are able to show the interesting fact that $U_{\lambda}$ may be chosen once and for all so that (1.3) remains valid for each $r=1,2, \cdots, n$.

We proceed to the development of these results now.

2. Preliminaries. Throughout, $\mathscr{C}$ will be a Hilbert space (finite or infinite dimensional) with inner product $\langle\cdot, \cdot\rangle$. For each $r=1,2, \cdots$, we construct the vector space

$$
\Lambda^{r} \mathscr{H}=\operatorname{sp}\left[\left(x_{1} \wedge x_{2} \wedge \cdots \wedge x_{r}\right)\right], x_{1}, x_{2}, \cdots, x_{r} \in \mathscr{C}
$$

spanned by all decomposable $r$-vectors $x_{1} \wedge x_{2} \wedge \cdots \wedge x_{r}$, where the vectors $x_{1}, x_{2}, \cdots, x_{r}$ run over $\mathscr{H}$. We use the wedge $\wedge$ to deonte the exterior (Grassmann) product and the symbol

$$
\operatorname{sp}[x], x \in \mathscr{A}
$$

denotes the vector subspace generated by all $x$ in $\mathscr{A}$. (See Vala [12].)

An inner product may be defined on $\Lambda^{r} \mathscr{H}$ by requiring that for decomposable vectors $x_{1} \wedge x_{2} \wedge \cdots \wedge x_{r}$, and $y_{1} \wedge y_{2} \wedge \cdots \wedge y_{r}$ in $\Lambda^{r} \mathscr{H}$, 


$$
\left\langle x_{1} \wedge x_{2} \wedge \cdots \wedge x_{r}, y_{1} \wedge y_{2} \wedge \cdots \wedge y_{r}\right\rangle=\operatorname{det}\left(\left\langle x_{i}, y_{j}\right\rangle\right),
$$

the determinant of the $r \times r$ matrix with $i j^{\text {th }}$ entry $\left\langle x_{i}, y_{i}\right\rangle$. (See [8, Ch. XVI].)

Let $A$ be any bounded linear operator on $\mathscr{H}$. Then a (bounded) linear operator $C_{r}(A)$, the $r^{\text {th }}$ compound of $A$, is defined on $A^{r} \mathscr{H}$ by the equation

$$
C_{r}(A)\left(x_{1} \wedge x_{2} \wedge \cdots \wedge x_{r}\right)=A x_{1} \wedge A x_{2} \wedge \cdots \wedge A x_{r},
$$

for all $x_{1}, x_{2}, \cdots, x_{r}$ in $\mathscr{X}$.

As a notational convenience, we shall introduce $Q_{n, r}$, the $\left(\begin{array}{l}n \\ r\end{array}\right)$ element set of order-preserving functions $\sigma$ sending $\{1,2, \cdots, r\}$ into $\{1,2, \cdots, r, \cdots n\}$, where $1 \leqq r \leqq n$. More exactly,

$$
Q_{n, r}=\{\sigma:\{1,2, \cdots, r\} \longrightarrow\{1,2, \cdots, r, \cdots, n\}\}
$$

where $1 \leqq \sigma(1)<\sigma(2)<\cdots<\sigma(r) \leqq n$.

As an immediate use of this set $Q_{n, r}$, we set down the following useful property:

Proposition 2.1. Let $M$ be an n-dimensional subspace of $\mathscr{H}$ having o.n. basis $\mathscr{E}=\left\{e_{1}, e_{2}, \cdots, e_{n}\right\}$. Then for each integer $r$, $1 \leqq r \leqq n$, the $\left(\begin{array}{l}n \\ r\end{array}\right)$-element set

$$
\mathscr{E}_{r}=\left\{e_{\sigma(1)} \wedge e_{\sigma(2)} \wedge \cdots \wedge e_{\sigma(r)}: \sigma \in Q_{n, r}\right\}
$$

is o.n. in $\Lambda^{r} \mathscr{H}$. Moreover, if $M$ is the orthogonal projection on $\mathscr{H}$ with range $M$ spanned by $\mathscr{E}$, then $C_{r}(M)$ is the orthogonal projection on $\Lambda^{r} \mathscr{H}$ with range spanned by the o.n. set $\mathscr{E}_{r}$.

Proof. The fact that $\mathscr{E}_{r}$ is o.n. in $\Lambda^{r} \mathscr{H}$ is immediate from (2.1). Similarly, by extending $\mathscr{E}$ to an o.n. basis for all of $\mathscr{H}$, the second assertion of our proposition follows from (2.1) and (2.2). (See also de Pillis [3].) This ends the proof.

Given the $n$-element set $\left\{x_{1}, x_{2}, \cdots, x_{n}\right\}$ in $\mathscr{H}$. Then for each $\sigma \in Q_{n r}, x_{\sigma}$ will be that vector in $\Lambda^{r} \mathscr{H}$ defined by

$$
\underline{x}_{\sigma}=x_{\sigma(1)} \wedge x_{\sigma(2)} \wedge \cdots \wedge x_{\sigma(r)} .
$$

Now let $A$ be any bouded linear operator on $\mathscr{H}$. Let $M$ be (the orthogonal projection onto) a finite dimensional subspace of $\mathscr{H}$. Then for each $r=1,2, \cdots, n E_{r}(A M)$, the $r^{\text {th }}$ elementary symmetric function of $A M$ is defined by

$$
E_{r}(A M)=\operatorname{tr}\left(C_{r}(A M)\right),
$$

the trace of the operator $C_{r}(A M)$ on $A^{r} \mathscr{H}$. 
The existence of the trace presents no problem since $M$ is finite dimensional (hence, $C_{r}(A M)$ is finite dimensional on $\Lambda^{r} \mathscr{H}$ ). In fact we offer the following more explicit form for $E_{r}(A M)$ :

Proposition 2.2. For $A$ a bounded linear operator on $\mathscr{H}$, and for $M$ an n-dimensional subspace with o.n. basis $\left\{e_{1}, e_{2}, \cdots, e_{n}\right\}$, we have the equality

$$
E_{r}(A M)=\sum_{\sigma \in Q_{n}, r}\left\langle C_{r}(A M) \underset{\sim}{e_{\sigma}},{\underset{\sim}{\sigma}}_{\sigma}\right\rangle
$$

Proof. The trace of any bounded operator $B$ on Hilbert space may be written as

$$
\sum_{i \in I}\left\langle B e_{i}, e_{i}\right\rangle \text { where }\left\{e_{i}\right\}_{i \in I}
$$

is any o.n. basis of $\mathscr{l}$. Since $C_{r}(A M)=C_{r}(A) C_{r}(M)$ (see 2.2)) is zero on all but a finite-dimensional subspace $C_{r}(M)$ of $\Lambda^{r} \mathscr{H}$, it suffices to consider the sum above relative to the finite o.n. basis $\left\{e_{\sigma}: \sigma \in Q_{n, r}\right\}$ of $C_{r}(M)$ (see Porposition 2.1). That is,

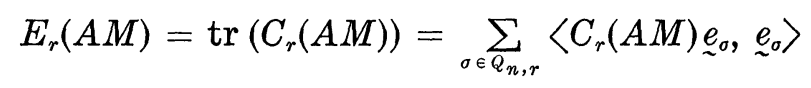

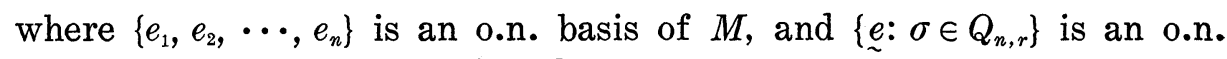
basis of $C_{r}(M)$. The proof is done.

REMARK. It is to be noted that

$$
E_{r}(A M)=\sum_{\sigma \in Q_{n}, r}\left\langle C_{r}(A M) \underset{\sim}{e_{\sigma}}, \underset{\sim}{e_{\sigma}}\right\rangle=\sum_{\sigma \in Q_{n}, r}\left\langle C_{r}(A) \underset{\sim}{e_{\sigma}},{\underset{\sim}{e}}_{\sigma}\right\rangle,
$$

so that the appearance of $M=\operatorname{sp}\left[e_{1}, e_{2}, \cdots e_{n}\right]$ becomes superfluous in (2.5). To see this, observe that $C_{r}(A M)=C_{r}(A) C_{r}(M)$ (from (2.2)), and that $C_{r}(M) \underset{\sim}{e_{\sigma}}={\underset{\sim}{\sigma}}_{\sigma}$.

REMARK. Let us write $\operatorname{tr}(A M)$ in the form

$$
\operatorname{tr}(A M)=\sum_{i=1}^{n}\left\langle A e_{i}, e_{i}\right\rangle
$$

where $\left\{e_{1}, e_{2}, \cdots, e_{n}\right\}$ is any o.n. basis of $M$. Then the extended Hausdorff-Toeplitz theorem of Berger [1], [6] tells us that the set $W_{n}(A)$ of all such $n$-term sums, is convex. Can we then conclude that the set $W_{r, n}(A)$ of all $\left(\begin{array}{l}n \\ r\end{array}\right)$-term sums (2.6) is also convex (by replacing $A$ in (2.7) with $C_{r}(A)$ and replacing $e_{i}$ with $\left.e_{\sigma}\right)$ ? The answer is the convexity $W_{r, n}(A)$ cannot be so deduced from the convexity of $W_{n}(A)$ since in (2.7), all $n$-element o.n. sets $\left\{e_{1}, e_{2}, \cdots, e_{n}\right)$ of $\mathscr{C}$ are 
permitted. Contrariwise, in considering $W_{r, n}(A)$ given in (1.2), the set of all sums of the form (2.6), only those o.n. sets $\left\{{\underset{\sim}{1}}_{1}, \underline{\sim}_{2}, \cdots, \underline{e}_{R}\right\}$, $R=\left(\begin{array}{l}n \\ r\end{array}\right)$, are permitted where each $e_{i}$ is a decomposable unit vector of $\Lambda^{r} \mathscr{H}$. Recall that $\Lambda^{r} \mathscr{H}$ is the span of all decomposable vectors $x_{1} \wedge x_{2} \wedge \cdots \wedge x_{r}$ so that in general, not every unit vector is decomposable.

Now let $M$ be an $r$-dimensional subspace of $\mathscr{H}$ with o.n. basis $\left\{e_{1}, e_{2}, \cdots, e_{r}\right\}$. Let $A$ be a bounded linear operator on $\mathscr{H}$ and let $z$ be complex. Then

$$
\left\langle C_{r}(A+z) e_{1} \wedge e_{2} \wedge \cdots \wedge e_{r}, e_{1} \wedge e_{2} \wedge \cdots e_{r}\right\rangle
$$

is the $r^{\text {th }}$ degree polynomial

$$
\sum_{j=0}^{r} \sum_{\sigma \in Q_{r, j}} z^{r-j}\left\langle C_{j}(A){\underset{\sim}{\sigma},}_{e_{o}}\right\rangle:
$$

For $j=0$, we define $\sum_{\sigma \in Q_{r, 0}}\left\langle C_{0}(A) \underset{\sim}{e_{\sigma}}, \underset{\sim}{\left.e_{\sigma}\right\rangle}\right.$ to be the number 1 . The equivalence of (2.8)' and (2.8) follows from the orthogonality of the set $\left\{e_{1}, e_{2}, \cdots, e_{r}\right\}$ along with use of (2.1) and (2.2).

Finally, we define Berger bases for a pair of $r$-dimensional subspaces $M$ and $N$ in $\mathscr{H}$, after C. A. Berger, who proved [1], [6], that such pairs of bases always exist for any such $M$ and $N$.

Definition 2.3. The orthonormal bases $\left\{x_{1}, x_{2}, \cdots, x_{r}\right\} \subset M$ and $\left\{y_{1}, y_{2}, \cdots, y_{r}\right\} \subset N$ will be called Berger bases (relative to $M$ and $N$ ) if and only if $\left\langle x_{i}, y_{j}\right\rangle=0$ whenever $i \neq j$. (Note: No constraint is placed on $\left\langle x_{i}, y_{i}\right\rangle$.)

3. Convergence properties of $A$. In what follows, $A$ will be a fixed bounded linear operator on Hilbert space $\mathscr{H}$ and $x$ is a unit vector in $\mathscr{H}$.

DEFINITION 3.1. The unit vector $x_{z} \in \mathscr{H}$ is defined for each sufficiently large complex $z$ by the conditions that

$$
(A+z) x_{z}=\left\langle(A+z) x_{z}, x\right\rangle x,
$$

and

$$
\left\langle x_{z}, x\right\rangle>0
$$

REMARK. Since $A$ is bounded, sufficiently large complex $z$ may always be found so that $A+z$ is invertible; that is, so that a unit vector $x_{z}$ always exists which is sent by $A+z$ to a scalar multiple of the fixed unit vector $x$. We note that if Condition (1) above obtains for some unit vector $x_{z}$, then it obtains equally well for the 
vector $w \cdot x_{z}$, where $\underline{w}$ is any complex scalar of modulus one. Hence, Condition (2) is presented to allow a unique $x_{z}$; as we shall now see, $x_{z}$ has the further property that $x_{z} \rightarrow x$ as $z \rightarrow \infty$. In fact, we present a stronger statement of convergence in the following lemma:

LEMMA 3.2. Given the bounded linear operator $A$ and unit vectors $x$ and $x_{z}$ defined in Definition 3.1. Then

$$
z\left(1-\left|\left\langle x_{z}, x\right\rangle\right|^{2}\right) \longrightarrow 0 \text { as } z \longrightarrow \infty \text {. }
$$

Consequently, $x_{z} \rightarrow x$ as $z \rightarrow \infty$.

Proof. We write the expression $\left\langle(A+z) x_{z}, x_{z}\right\rangle$ in two ways:

$$
\left\langle(A+z) x_{z}, x_{z}\right\rangle=\left\langle A x_{z}, x_{z}\right\rangle+z \text {. }
$$

Substituting $\left\langle(A+z) x_{z}, x\right\rangle x$ for $(A+z) x_{z}$, yields

$$
\left\langle(A+z) x_{z}, x_{z}\right\rangle=\left\langle A x_{z}, x\right\rangle\left\langle x, x_{z}\right\rangle+z\left|\left\langle x_{z}, x\right\rangle\right|^{2} .
$$

Subtraction of (3.2) from (3.1) gives us

$$
z\left(1-\left|\left\langle x_{z}, x\right\rangle\right|^{2}\right)=\left\langle A x_{z}, x\right\rangle\left\langle x, x_{z}\right\rangle-\left\langle A x_{z}, x_{z}\right\rangle .
$$

From (3.3) it follows that $\left\langle x_{z}, x\right\rangle \rightarrow 1$ as $z \rightarrow \infty$; to see this, divide through by $z$ and let $z$ go to infinity. This, in turn, implies that

$$
x_{z} \longrightarrow x \text { as } z \longrightarrow \infty \text {. }
$$

Again from (3.3) we may deduce that $z\left(1-\left|\left\langle x_{z}, x\right\rangle\right|^{2}\right) \rightarrow 0$ as $z \rightarrow \infty$; use the continuity of both $A$ and the inner product along with (3.4) to show that the right-hand side of (3.3) goes to zero as $z \rightarrow \infty$. The lemma is proved.

For later convenience, the following notations are introduced:

Definition 3.3. Let $\left\{x_{1}, x_{2}, \cdots, x_{r}\right\}$ be any finite set spanning subspace $M$ of Hilbert space $\mathscr{H}$. Let $A$ be the compression to $M$ of some bounded operator on $\mathscr{H}$. (That is, $A=P_{M} A P_{M}$, where $P_{M}$ is the orthogonal projection onto $M$.) $B y x_{z}$, we mean that unit vector of $M$ defined in Definition 3.1 relative to $x_{r}$. That is,

and

$$
(A+z) x_{z}=\left\langle(A+z) x_{z}, x_{r}\right\rangle x_{r},
$$

$$
\left\langle x_{z}, x_{r}\right\rangle>0 \text {. }
$$

We then define the vectors $x_{z}$ in $\Lambda^{r} \mathscr{H}$, and $x_{j}$ in $\Lambda^{j} \mathscr{H}$, for $j=$ $1,2, \cdots, r$ by the following equations:

$$
\underline{x}_{z}=x_{1} \wedge x_{2} \wedge \cdots \wedge x_{r-1} \wedge x_{z},
$$


and

$$
{\underset{x}{j}}_{j}=x_{1} \wedge x_{2} \wedge \cdots \wedge x_{j}, \quad \text { for each } j=1,2, \cdots, r .
$$

We shall have occasion to use the notion of an operator $A$ having power $r$ on a vector $x$, the definition of which follows now.

Definition 3.4. Let $A$ be a bounded linear operator on Hilbert space $M$. Let $x$ be a unit vector of $M$ and let $x_{z}$ be the unit vector defined above (Definition 3.1). Then $A$ is said to have power $r$ on $x$ if

$$
\lim _{z \rightarrow \infty} z^{r}\left(1-\left\langle x_{z}, x\right\rangle^{2}\right)=0 .
$$

A simple restatement of Lemma 3.2, using the terminology of Definition 3.4, is the proposition:

Proposition 3.5. For any bounded linear operator $A$ on $\mathscr{H}$ and for any unit vector $x \in \mathscr{H}, A$ has power one on $x$.

One important instance occurs where $A$ has power $r$ on $x$ for all integer values of $r=1,2, \cdots$. Specifically, we have

Proposition 3.6. Suppose $x$ is an eigenvector for $A$. Then $A$ has unbounded power on $x$. That is, for all $r=1,2, \cdots, m, \cdots$,

$$
z^{r}\left(1-\left|\left\langle x_{z}, x\right\rangle\right|^{2}\right) \longrightarrow 0 \text {. }
$$

Proof. Observe that for all $z, x_{z}=x$ whenever $x$ is an eigenvector of $A$.

4. Induction hypothesis. In the following section, we shall refer to and hence extend the induction hypothesis which we now present.

Induction Hypothesis. Given a bounded linear operator $A$ on $\mathscr{H}$. Given $n$-dimensional subspaces $M$ and $N$ of $\mathscr{H}$ with Berger bases $\left\{x_{1}, x_{2}, \cdots, x_{n}\right\}$ and $\left\{y_{1}, y_{2}, \cdots, y_{n}\right\}$, respectively. Let the o.n. set $\left\{u_{1}, u_{2}, \cdots, u_{n}\right\}$ be defined by requiring of each $u_{i} \in \operatorname{sp}\left[x_{i}, y_{i}\right]$, that

$$
\lambda\left\langle A x_{i}, x_{i}\right\rangle+(1-\lambda)\left\langle A y_{i}, y_{i}\right\rangle=\left\langle A u_{i}, u_{i}\right\rangle
$$

for arbitrary but fixed $\lambda \in(0,1)$. Then

$$
\lambda\left\langle C_{j}(A){\underset{\sim}{x}}_{\sigma}, x_{\sigma}\right\rangle+(1-\lambda)\left\langle C_{j}(A) \underline{\sim}_{\sigma}, \underline{\sim}_{\sigma}\right\rangle=\left\langle C_{j}(A) \underline{\sim}_{\sigma}, \underline{\sim}_{\sigma}\right\rangle
$$

for each $j=1,2, \cdots, r-1$, and for each $\sigma \in Q_{n j}$. 
REMARK 4.1. In the "earliest" possible case, $j=1, r=2$, the induction hypothesis reduces to the classical Hausdorff-Toeplitz theorem relative to the Berger bases for $M$ and $N$. In what follows, we shall prove that under certain restrictions on $A$, or on the subspaces $M$ and $N$, the induction hypothesis may be extended from the cases $j=1,2, \cdots, r-1$ to the cases $j=1,2, \cdots, r-1, r$.

REMARK 4.2. The fixed o.n. sets $\left\{x_{i}\right\},\left\{y_{i}\right\}$ and $\left\{u_{i}\right\}$ meet all the conditions described above if the operator $A$ is replaced by $A+z$, where $z$ is any complex scalar (i.e., $z$ is a complex scalar multiple of the identity operator).

5. A quasi-convexity result. We now extend our induction hypothesis to a convexity result involving the operator $A+z$ (Theorem 5.2).

REMARK 5.1. Let $F$ be some scalar-valued function on the unit sphere of $\mathscr{H}$, which has convex range. That is, for all $\lambda \in(0,1)$, and for all $x, y$ in $\mathscr{H}$ such that $\|x\|=\|y\|=1$, there exists $u$, $\|u\|=1$ such that

$$
\lambda F(x)+(1-\lambda) F(y)=F(u) .
$$

Now let $p_{1}(\theta, z)$ and $p_{2}(\theta, z)$ be two complex valued functions of $z$, whose values have the same argument $\theta$ for each $z$; the common argument, $\theta$, necessarily depends on $z$. Then

$$
\lambda p_{1}(\theta, z) F(x)+(1-\lambda) p_{2}(\theta, z) F(y)=\left(\lambda p_{1}(\theta, z)+(1-\lambda) p_{2}(\theta, z)\right) F\left(u_{z}\right),
$$

where $u_{z}$ is a unit vector depending on $z$.

To verify (5.2), divide both sides by $\lambda p_{1}(\theta, z)+(1-\lambda) p_{2}(\theta, z)$. The fact that $p_{1}(\theta, z)$ and $p_{2}(\theta, z)$ each factor as $e^{i \theta}$ times positive scalars, reveals the lefthand side of (5.2) as a convex combination of $F(x)$ and $F(y)$.

THEOREM 5.2. (A quasi convexity theorem) Suppose the induction hypothesis obtains for each $j=1,2, \cdots, r-1$. Let $M$ and $N$ be $r$ dimensional subspaces of $\mathscr{H}$ with Berger bases $\left\{x_{1}, x_{2}, \cdots, x_{r}\right\}$ and $\left\{y_{1}, y_{2}, \cdots, y_{r}\right\}$, respectively. Then for every neighborhood $U(\infty)$ of infinity, there exists $a z \in U(\infty)$ such that

$$
\lambda\left\langle C_{r}(A+z) \underset{\sim}{x_{z}}, \underset{\sim}{x_{z}}\right\rangle+(1-\lambda)\left\langle C_{r}(A+z){\underset{\sim}{z}}_{z}, \underline{\sim}_{z}\right\rangle=\left\langle C_{r}(A+z) \underset{\sim}{u_{z}},{\underset{\sim}{u}}_{z}\right\rangle
$$

where $x_{z}$ and $\underline{y}_{z}$ are defined above (Definition 3.3) and 


$$
\underline{u}_{z}=u_{1} \wedge u_{2} \wedge \cdots \wedge u_{r-1} \wedge u_{z},
$$

where $u_{z}$ is a unit vector of $\mathrm{sp}\left[x_{z}, y_{z}\right]$.

Proof. Recall that $x_{z}$ is that unit vector such that

$$
(A+z) x_{z} \in \operatorname{sp}\left[x_{r}\right]
$$

where $\left\langle x_{z}, x_{r}\right\rangle>0$. (Definition 3.3.) Thus, $(A+z) x_{z}$ is orthogonal to $\mathrm{sp}\left[x_{1}, x_{2}, \cdots, x_{r-1}\right]$. From the definition (2.1) of the inner product on $\Lambda^{r} \mathscr{H}$, we have

$$
\begin{aligned}
& \lambda\left\langle C_{r}(A+z) \underset{\sim}{x_{z}}, \underset{\sim}{x_{z}}\right\rangle \\
(5.3)= & \left.\lambda(A+z) x_{1} \wedge \cdots \wedge(A+z) x_{r-1} \wedge(A+z) x_{z}, x_{1} \wedge \cdots \wedge x_{r-1} \wedge x_{z}\right\rangle \\
= & \lambda\left\langle C_{r-1}(A+z){\underset{\sim}{r-1}}_{x_{r-1}}{\underset{\sim}{r-1}}_{r}\right\rangle\left\langle(A+z) x_{z}, x_{z}\right\rangle .
\end{aligned}
$$

Similarly, we may write

$$
(1-\lambda)\left\langle C_{r}(A+z){\underset{\sim}{z}}_{z},{\underset{\sim}{z}}_{z}\right\rangle=(1-\lambda)\left\langle C_{r-1}(A+z){\underset{\sim}{r-1}}_{\underbrace{}_{r-1}}{\underset{\sim}{r}}_{r}\right\rangle\left\langle(A+z) y_{z}, y_{z}\right\rangle .
$$

We add (5.3) and (5.4) to obtain

$$
\begin{aligned}
& \lambda\left\langle C_{r}(A+z){\underset{\sim}{z}}_{z},{\underset{\sim}{x}}_{z}\right\rangle+(1-\lambda)\left\langle C_{r}(A+z){\underset{\sim}{z}}_{z},{\underset{\sim}{z}}_{z}\right\rangle \\
= & \lambda\left\langle C_{r-1}(A+z){\underset{r}{r-1}}_{x_{r-1}}\right\rangle\left\langle(A+z) x_{z}, x_{z}\right\rangle \\
& +(1-\lambda)\langle C_{r-1}(A+z){\underset{\sim}{r-1}}_{r}, \underbrace{y_{r-1}}_{r-1}\rangle\left\langle(A+z) y_{z}, y_{z}\right\rangle .
\end{aligned}
$$

We assert that for each neighborhood $U(\infty)$ of infinity, a $z \in U(\infty)$ may be found so that the (complex) arguments of the two monic $(r-1)$ st degree polynomials,

$$
\left\langle C_{r-1}(A+z) \underset{\sim}{x_{r-1}},{\underset{\sim}{x-1}}_{r-1}\right\rangle \text { and }\left\langle C_{r-1}(A+z){\underset{\sim}{r-1}}_{r},{\underset{\sim}{r-1}}_{y_{r-1}}\right\rangle
$$

(see (2.8)), agree. In fact, their quotient, call it $f$, is an analytic function in a neighborhood of infinity; moreover, $f(z)$ converges to one at infinity. By the open mapping theorem for analytic functions (c.f. [2: pg 175], [7]), open neighborhoods (of analyticity) of infinity will be sent by $f$ to open neighborhoods of the positive number one. Therefore, every neighborhood of infinity will be sent by $f$ to open neighborhoods of the positive number one. Thus, every neighborhood of infinity contains a $z$ such that $f(z)>0$; moreover, $f(z)$ is as near to one as we please. That is, every neighborhood $U(\infty)$ of infinity contains a $z$ for which the arguments of

$$
\left\langle C_{r-1}(A+z){\underset{\sim}{r-1}}_{x_{r-1}}\right\rangle \text { and }\left\langle C_{r}(A+z){\underset{\sim}{r-1}}_{x_{r-1}} \underline{\sim}_{r-1}\right\rangle
$$

are equal. Replace the symbols $p_{1}(\theta)$ and $p_{2}(\theta)$ in (5.2), Remark 5.1, by the polynomials of (5.6), and replace $F(x)$ and $F(y)$ by $\left\langle(A+z) x_{z}, x_{z}\right\rangle$ 
and $\left\langle(A+z) y_{z}, y_{z}\right\rangle$, to obtain the following equivalent expression for the right hand side of (5.5):

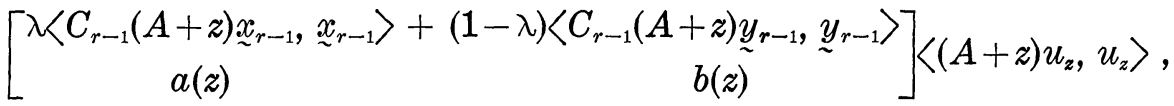

$$
\begin{aligned}
& =[a(z)+b(z)]\left\langle(A+z) u_{z}, u_{z}\right\rangle
\end{aligned}
$$

where $u_{z}$ in $\operatorname{sp}\left[x_{z}, y_{z}\right]$ is a unit vector satisfying the equation

$$
\frac{a(z)}{a(z)+b(z)}\left\langle(A+z) x_{z}, x_{z}\right\rangle+\frac{b(z)}{b(z)+b(z)}\left\langle(A+z) y_{z}, y_{z}\right\rangle=\left\langle(A+z) u_{z}, u_{z}\right\rangle .
$$

This, in turn, is equivalent to the equation

$$
\frac{a(z)}{a(z)+b(z)}\left\langle A x_{z}, x_{z}\right\rangle+\frac{b(z)}{a(z)+b(z)}\left\langle A y_{z}, y_{z}\right\rangle=\left\langle A u_{z}, u_{z}\right\rangle
$$

for $a(z)$ and $b(z)$ defined in (5.7).

We note that the quotients $a(z) /(a(z)+b(z))$ and $b(z) /(a(z)+b(z))$ approach the values $\lambda$ and $(1-\lambda)$, respectively as $z \rightarrow \infty$. (To see this, divide numerators and denominators by $z^{r-1}$ and pass to the limit as $z \rightarrow \infty$.) Since $x_{z} \rightarrow x_{r}$ and $y_{z} \rightarrow y_{r}$ (Lemma 3.2) as $z \rightarrow \infty$, we have

$$
\left\langle A u_{z}, u_{z}\right\rangle \longrightarrow\left\langle A u_{r}, u_{r}\right\rangle \text { as } z \longrightarrow \infty \text {. }
$$

(Recall that $u_{r}$ is a unit vector of $\mathrm{sp}\left[x_{r}, y_{r}\right]$ satisfying the equation $\lambda\left\langle A x_{r}, x_{r}\right\rangle+(1-\lambda)\left\langle A y_{r}, y_{r}\right\rangle=\left\langle A u_{r}, u_{r}\right\rangle$.)

We couple the statement of our induction hypothesis (see (2.8) and Remark 4.2) with (5.7) to replace the left-hand factor of (5.7) by the $r-1$ degree polynomial

(5.10) $\left\langle C_{r-1}(A+z) u_{1} \wedge \cdots \wedge u_{r-1}, u_{1} \wedge \cdots \wedge u_{r-1}\right\rangle=\left\langle C_{r-1}(A+z){\underset{\sim}{r-1}}_{r}, \underline{\sim}_{r-1}\right\rangle$,

where each unit vector $u_{i}$ in $\operatorname{sp}\left[x_{i}, y_{i}\right], i=1,2, \cdots, r-1$ has the property that

$$
\lambda\left\langle A x_{i}, x_{i}\right\rangle+(1-\lambda)\left\langle A y_{i}, y_{i}\right\rangle=\left\langle A u_{i}, u_{i}\right\rangle .
$$

This yields the simplified form

$$
\left\langle C_{r-1}(A+z) \underline{\sim}_{r-1}, \underline{\sim}_{r-1}\right\rangle\left\langle(A+z) u_{z}, u_{z}\right\rangle
$$

which is equivalent to (5.5) and to (5.7).

Notice that $(A+z) u_{z}$ is a certain linear combination of the vectors $x_{r}$ and $y_{r}$. This is so because $u_{z} \in \mathrm{sp}\left[x_{z}, y_{z}\right]$ and $(A+z)$ sends $x_{z}$ into $\mathrm{sp}\left[x_{r}\right]$ and also sends $y_{z}$ into $\mathrm{sp}\left[y_{r}\right]$ by definition (Definitions 3.1 and 3.3). Thus, $(A+z) u_{z}$ is orthogonal to $\operatorname{sp}\left[u_{1}, u_{2}, \cdots, u_{r-1}\right]$; 
orthogonality is guaranteed by the fact that

$$
\left\{x_{1}, x_{2}, \cdots, x_{r}\right\} \text { and }\left\{y_{1}, y_{2}, \cdots, y_{r}\right\}
$$

are Berger bases for $M$ and $N$, respectively (Definition 2.3). Accordingly, we may write (5.11) as follows:

$$
\left\langle C_{r}(A+z) u_{1} \wedge \cdots \wedge u_{r-1} \wedge u_{z}, u_{1} \wedge \cdots \wedge u_{r-1} \wedge u_{z}\right\rangle=\left\langle C_{r}(A+z) \underline{\sim}_{z}, \underline{u}_{z}\right\rangle .
$$

If we combine (5.12) with (5.5), we obtain the final equality

$$
\lambda\left\langle C_{r}(A+z){\underset{\sim}{z}}_{z},{\underset{\sim}{z}}_{z}\right\rangle+(1-\lambda)\left\langle C_{r}(A+z) \underline{v}_{z}, \underset{\sim}{y_{z}}\right\rangle=\left\langle C_{r}(A+z) \underset{\sim}{u_{z}},{\underset{\sim}{u}}_{z}\right\rangle .
$$

Statement (5.13) above completes the proof of the theorem.

6. A convexity result for $W_{r, n}(A)$. In this section, we combine the quasi-convexity result (Theorem 5.2) with the notion of $A$ having sufficient integer power on certain $x$ (Definition 3.4) to obtain a convexity theorem for $A$ (Theorem 6.1). As a consequence, we obtain our main convexity result (Theorem 6.2) which holds for arbitrary but fixed linear operator $A$ so long as the subspace $M$ and $N$ are mutually orthogonal.

THEOREM 6.1. Let $M$ and $N$ be r-dimensional subspaces of Hilbert space $\mathscr{H}$ having o.n. Berger bases $\left\{x_{1}, x_{2}, \cdots, x_{r}\right\}$ and $\left\{y_{1}, y_{2}, \cdots, y_{r}\right\}$, respectively. Suppose the bounded linear operator $A$, when restricted to each of these subspaces has power $r$ on $x_{r}$ and $y_{r}$; that is,

$$
\lim _{z \rightarrow \infty} z^{r}\left(1-\left|\left\langle x_{z}, x_{r}\right\rangle\right|^{2}\right)=\lim _{z \rightarrow \infty} z^{r}\left(1-\left|\left\langle y_{z}, y_{r}\right\rangle\right|^{2}\right)=0 \text {. }
$$

If an o.n. set $\left\{u_{1}, u_{2}, \cdots, u_{r}\right\}$ satisfies the equations

$$
\lambda\left\langle A x_{i}, x_{i}\right\rangle+(1-\lambda)\left\langle A y_{i}, y_{i}\right\rangle=\left\langle A u_{i}, u_{i}\right\rangle, i=1,2, \cdots, r .
$$

for arbitrary but fixed $\lambda \in(0,1)$, then necessarily,

$$
\lambda\left\langle C_{r}(A) \underline{x}_{r}, \underline{\sim}_{r}\right\rangle+(1-\lambda)\left\langle C_{r}(A) \underline{\sim}_{r}, \underline{\sim}_{r}\right\rangle=\left\langle C_{r}(A){\underset{\sim}{u}}_{r}, \underline{\sim}_{r}\right\rangle
$$

whenever

$$
\lim _{z \rightarrow \infty} z^{r}\left(1-\left.\left\langle u_{z}, u_{r}\right\rangle\right|^{2}\right)=0
$$

where the unit vector $u_{z}$ satisfies the equation

$$
\lambda\left\langle A x_{z}, x_{z}\right\rangle+(1-\lambda)\left\langle A y_{z}, y_{z}\right\rangle=\left\langle A u_{z}, u_{z}\right\rangle .
$$


Proof. Consider the function

$$
\varphi(z)=\lambda\left\langle C_{r}(A+z) \underline{x}_{z}, z_{z}\right\rangle+(1-\lambda)\left\langle C_{r}(A+z) \underline{\sim}_{z}, \underline{\sim}_{z}\right\rangle-\left\langle C_{r}(A+z) \underline{u}_{z}, \underline{u}_{z}\right\rangle .
$$

Now $x_{z} \in \operatorname{sp}\left[x_{1}, x_{2}, \cdots, x_{r}\right]$ must be of the form

$$
x_{z}=\sum_{i=1}^{r-1} a_{i} x_{i}+\left\langle x_{z}, x_{r}\right\rangle x_{r}
$$

for certain scalars $a_{1}, a_{2}, \cdots, a_{r-1}$. Thus

$$
\begin{aligned}
\underline{x}_{z} & =x_{1} \wedge \cdots \wedge x_{r-1} \wedge x_{z} \\
& =\sum_{i=1}^{r-1} a_{i}\left(x_{1} \wedge \cdots \wedge x_{1-1} \wedge x_{i}\right)+\left\langle x_{z}, x_{r}\right\rangle\left(x_{1} \wedge \cdots \wedge x_{r-1} \wedge x_{r}\right) \\
& =0+\left\langle x_{z}, x_{r}\right\rangle \underline{\sim}_{r},
\end{aligned}
$$

where each term in the summation involving $a_{1}, a_{2}, \cdots, a_{r-1}$ equals zero due to repetitions of $x_{i}$ in the Grassmann product

$$
x_{1} \wedge \cdots \wedge x_{r-1} \wedge x_{i} \text {. }
$$

We substitute $\left\langle x_{z}, x_{r}\right\rangle \underline{x}_{r}$ for $\underline{x}_{z}$ into our expression for $\phi(z)$ to obtain

$\varphi(z)=\lambda\left\langle C_{r}(A+z) \underline{x}_{r}, \underline{x}_{r}\right\rangle \alpha_{z}+(1-\lambda)\left\langle C_{r}(A+z){\underset{\sim}{y}}_{r}, \underline{\sim}_{r}\right\rangle \beta_{z}-\left\langle C_{r}(A+z) \underline{\sim}_{r}, \underline{\sim}_{r}\right\rangle \gamma_{z}$ where

$$
\alpha_{z}=\left|\left\langle x_{z}, x_{r}\right\rangle\right|^{2}, \beta_{z}=\left|\left\langle y_{z}, y_{r}\right\rangle\right|^{2} \text { and } \gamma_{z}=\left|\left\langle u_{z}, u_{r}\right\rangle\right|^{2} \text {. }
$$

If we substitute the explicit polynomial expressions for each of the three inner products in (6.2) (see (2.8)), we obtain the following expression for $\varphi(z)$ :

$$
\sum_{j=0}^{r} \sum_{\sigma} z_{\varepsilon} z^{r-j},\left[\lambda\left\langle C_{j}(A) \underset{\sim}{x_{\sigma}},{\underset{\sim}{x}}_{\sigma}\right\rangle \alpha_{z}+(1-\lambda)\left\langle C_{j}(A) \underline{\sim}_{\sigma}, \underline{\sim}_{\sigma}\right\rangle \beta_{z}-\left\langle C_{j}(A) \underset{\sim}{u_{\sigma}}, \underline{\sim}_{\sigma}\right\rangle \gamma_{z}\right] .
$$

Our induction hypothesis allows us to replace $\left\langle C_{j}(A){\underset{\sim}{\sigma}}_{u^{\prime}}{\underset{\sim}{\sigma}}_{\sigma}\right\rangle$ in (6.4) by

$$
\lambda\left(C_{j}(A) \underset{\sim}{x_{\sigma}}, \underset{\sim}{x_{\sigma}}\right\rangle+(1-\lambda)\left\langle C_{j}(A) \underline{\sim}_{\sigma},{\underset{\sim}{\sigma}}_{\sigma}\right\rangle,
$$

at least for the cases $j=1,2, \cdots, \cdots, r-1$, and for all $\sigma \in Q_{r, j}$. Effecting this substitution, (6.4) yields the following form for $\varphi(z)$ :

$$
\begin{aligned}
\varphi(z)= & z^{r}\left[\lambda\left(\alpha_{z}-\gamma_{z}\right)+(1-\lambda)\left(\beta_{z}-\gamma_{z}\right]\right. \\
& +\sum_{j=1}^{r-1} z^{r-j}{\underset{\sim}{X}}_{j}\left(\alpha_{z}-\gamma_{z}\right)+\sum_{i=1}^{r-1} z^{r-j}{\underset{\sim}{j}}_{j}\left(\beta_{z}-\gamma_{z}\right) \\
& +\lambda\left\langle C_{r}(A) \underset{\sim}{x_{r}}, \underset{\sim}{\left.x_{r}\right\rangle \alpha_{z}+(1-\lambda)\left\langle C_{r}(A) \underline{\sim}_{r},{\underset{\sim}{r}}_{r}\right\rangle \beta_{z}-\left\langle C_{r}(A){\underset{\sim}{u}}_{r},{\underset{\sim}{u}}_{r}\right\rangle \gamma_{z},}\right.
\end{aligned}
$$


where for each $j=1,2, \cdots, r-1$,

$$
\underset{\sim}{X_{j}}=\lambda \sum_{\sigma \in Q_{r}, j}\left\langle C_{j}(A) \underline{x}_{\sigma},{\underset{x}{\sigma}}_{\sigma}\right\rangle \text {, and } \underline{\sim}_{j}=(1-\lambda) \sum_{\sigma \in Q_{r}, j}\left\langle C_{j}(A) \underline{y}_{\sigma}, \underline{y}_{\sigma}\right\rangle \text {. }
$$

Assumptions (i ) and (iii) together guarantee that for $k=1,2, \cdots, r$, the quantities

$$
z^{k}\left(\alpha_{z}-\gamma_{z}\right), \text { and } z^{k}\left(\beta_{z}-\gamma_{z}\right)
$$

tend to zero as $z \rightarrow \infty$. (Write $\alpha_{z}-\gamma_{z}=\left(1-\gamma_{z}\right)-\left(1-\alpha_{z}\right)$, and $\beta_{z}-\gamma_{z}=\left(1-\gamma_{z}\right)-\left(1-\beta_{z}\right)$.)

We then constrain the growth of $z$ in accordance with Theorem 5.2 , so that $\varphi(z)=0$ as $z \rightarrow \infty$. From hypothesis (i), $\alpha_{z}$ and $\beta_{z} \rightarrow 1$ as $z \rightarrow \infty$; from hypothesis (iii), $\gamma_{z} \rightarrow 1$ as $z \rightarrow \infty$. Thus, we may conclude that the "constant" term of $\varphi(z)$ (see (6.5)), which tends to

$$
\lambda\left\langle C_{r}(A){\underset{\sim}{r}}_{r}, \underline{\sim}_{r}\right\rangle+(1-\lambda)\left\langle C_{r}(A){\underset{\sim}{r}}_{r}, \underline{\sim}_{r}\right\rangle-\left\langle C_{r}(A){\underset{\sim}{r}}_{r}, \underline{u}_{r}\right\rangle,
$$

approaches the value zero. The proof of the theorem is done.

We now present our main result which, as a corollary to Theorem 6.1, holds for all bounded linear operators $A$ on $\mathscr{H}$, and all $r=$ $1,2, \cdots, n$, provided the $n$-dimensional subspaces $M$ and $N$ are orthogonal.

THeOREM 6.2. Let $M$ and $N$ be n-dimensional subspaces of $\mathscr{H}$ such that $M$ is orthogonal to $N$. Then for any bounded linear operator $A$, and for any $\lambda \in(0,1)$, there exists an $n$-dimensional subspace $U_{\lambda}$ in $M+N$ such that for each $r=1,2, \cdots, n$,

$$
\lambda E_{r}(A M)+(1-\lambda) E_{r}(A N)=E_{r}\left(A U_{\lambda}\right) .
$$

Note that $U_{\lambda}$ does not depend on $r$.

Proof. Let

$$
\mathscr{X}=\left\{x_{1}, x_{2}, \cdots, x_{r}, \cdots, x_{n}\right\}
$$

and

$$
\mathscr{Y}=\left\{y_{1}, y_{2}, \cdots, y_{r}, \cdots, y_{n}\right\}
$$

be o.n. bases of $M$ and $N$, respectively, which triangularize the compression operators $A: M \rightarrow M$ and $A: N \rightarrow N$. That is, let $x_{n}$ be an eigenvector for the (finitedimensional) compression of $A$ to $M$. Choose $x_{n-1}$ as a unit eigenvector of $A$ restricted to the orthogonal complement of $x_{n}$ in $M$; consequently, $A\left(x_{n-1}\right) \in \mathrm{sp}\left[x_{n-1}, x_{n}\right]$. Similarly, $x_{n-2}$ is a unit eigenvector of $A$ restricted to the orthogonal complement of $\operatorname{sp}\left[x_{n-1}, x_{n}\right]$, and so on, until for each $j=1,2, \cdots, n$, 


$$
A\left(x_{j}\right) \in \operatorname{sp}\left[x_{j}, x_{j+1}, \cdots, x_{n}\right] \text {. }
$$

By the same reasoning on $N,\left\{y_{1}, y_{2}, \cdots, y_{n}\right\}$ is an $0 . n$. basis of $N$ such that

$$
A\left(y_{j}\right) \in \mathrm{sp}\left[y_{j}, y_{j+1}, \cdots, y_{n}\right] \text {. }
$$

Due to the orthogonality of $M$ and $N, \mathscr{X}$ and $\mathscr{Y}$ are Berger bases. Moreover, corresponding $r$-element subsets $\left\{x_{\sigma(1)}, x_{\sigma(2)}, \cdots, x_{\sigma(r)}\right\}$ and $\left\{y_{\sigma(1)}, y_{\sigma(2)}, \cdots, y_{\sigma(r)}\right\}$ for each $\sigma \in Q_{n, r}$, are Berger bases for their respective linear spans, which we denote as follows:

$$
\begin{aligned}
M_{\sigma} & =\operatorname{sp}\left[x_{\sigma(1)}, x_{\sigma(2)}, \cdots, x_{\sigma(r)}\right] \\
N_{\sigma} & =\operatorname{sp}\left[y_{\sigma(1)}, y_{\sigma(2)}, \cdots, y_{\sigma(r)}\right]
\end{aligned}
$$

for each $\sigma \in Q_{n, r}$.

Note that $x_{\sigma(r)}$ and $y_{\sigma(r)}$ are eigenvectors for (the compression of) $A$ on the spaces $M_{\sigma}$ and $N_{\sigma}$, respectively: This follows from (6.6a) and (6.6b). Thus, $A$ has unbounded power on $x_{\sigma(r)}$ and $y_{\sigma(r)}$ when restricted to $M_{\sigma}$ and $N_{\sigma}$ (Proposition 3.6). Consequently, for $x_{z}$ and $y_{z}$ of Definition 3.3 (see also Lemma 3.2), $1-\left|\left\langle x_{z}, x_{\sigma(r)}\right\rangle\right|^{2}=1-\left|\left\langle y_{z}, y_{\sigma(r)}\right\rangle\right|^{2}=0$, since $x_{z}=x_{\sigma(r)}$ and $y_{z}=y_{\sigma(r)}$. Moreover, the $u_{z}$ defined in hypothesis (iv) of Theorem 6.1, can be chosen to equal $u_{\sigma(r)}$, where

$$
\lambda\left\langle A x_{\sigma(r)}, x_{\sigma(r)}\right\rangle+(1-\lambda)\left\langle A y_{\sigma(r)}, y_{\sigma(r)}\right\rangle=\left\langle A u_{\sigma(r)}, u_{\sigma(r)}\right\rangle .
$$

This implies that $z^{k}\left[1-\left|\left\langle u_{z}, u_{\sigma(r)}\right\rangle\right|^{2}\right]=0$ so that all hypothesis of Theorem 6.1 are fulfilled by each of the orthogonal subspaces $M_{o}$ and $N_{\sigma}$ as $\sigma$ runs over $Q_{n, r}$. Therefore, we may write for each $\sigma \in Q_{n, r}$, that

$$
\lambda\left\langle C_{r}(A) \underset{\sim}{x_{\sigma}},{\underset{\sim}{\sigma}}_{\sigma}\right\rangle+(1-\lambda)\left\langle C_{r}(A){\underset{\sim}{\sigma}}_{\sigma},{\underset{\sim}{\sigma}}_{\sigma}\right\rangle=\left\langle C_{r}(A) \underline{\sim}_{\sigma}, \underline{\sim}_{\sigma}\right\rangle,
$$

where $\left\{u_{1}, u_{2}, \cdots, u_{r}, \cdots, u_{n}\right\}$ is an $0 . \mathrm{n}$. set satisfying the equations

$$
\lambda\left\langle A x_{i}, x_{i}\right\rangle+(1-\lambda)\left\langle A y_{i}, y_{i}\right\rangle=\left\langle A u_{i}, u_{i}\right\rangle, i=1,2, \cdots, n .
$$

If we sum each side of (6.7) over all $\sigma \in Q_{n}$ (see Proposition 2.2 and (2.6)), we obtain

$$
\lambda E_{r}(A M)+(1-\lambda) E_{r}(A N)=E_{r}\left(A U_{\lambda}\right),
$$

where $U_{\lambda}=\operatorname{sp}\left[u_{1}, u_{2}, \cdots, u_{r}, \cdots, u_{n}\right]$. The theorem is proved.

REMARK. It is an open question as to whether $W_{r n}(A)$ ks always convex. It may be conjectured that if $2 r>\operatorname{dim} \mathscr{H}$, then convexity is automatic since, in this case, every vector of $A^{r} \mathscr{H}$ is automatically decomposable. (See Remarks following Proposition 2.2.) 


\section{REFERENCES}

1. C. A. Berger Normal dilations, Ph.D. Thesis, Cornell University, 1963.

2. H. Cartan, Theory of of analytic functions, Addison Wesley (1963).

3. J. de Pillis, Linear operators and their partitioned matrices Arch. der Math., 22 (1971), 79-84.

4. W. F. Donoghue, Jr., On the numerical range of a bounded operator, Mich. Math. J., (1957), 261-263.

5. P. R. Halmos, Numerical ranges and normal dilations, Acta Szeged, vol. 25, pp. $1-5$.

6. — A hilbert space problem book, Van Nostrand, Princeton, N. J. (1967).

7. M. Heins, Complex function theory, Academic Press (New York, 1968).

8. S. MacLane and G. Birkhoff, Algebra, Macmillan (New York, 1967).

9. R. Raghavendran, Toeplitz-Hausdorff theorem on numerical ranges, Proc. Amer. Math. Soc., 20 (1969), 214-285.

10. M. H. Stone, Hausdorff's theorem concerning Hermitian forms, Bull. Amer. Math. Soc., 36 (1930), 259-261.

11. R. C. Thompson, A note on normal matrices, Can. J. Math., 15 (1963), 220-225.

12. K. Vala, Sur la puissance exierieure d'un espace lineaire, Ann. Acad. Sci. Fenn. A. I. 233 (1956).

Received October 10, 1969 and in revised form September 23, 1970. Part of this work was performed under the auspices of the U.S. Atomic Energy Commission and part was supported by NSF Grant GP-8758.

BROOKHAVEN NATIONAL LABORATORY

UPTON, LONG ISLAND, NEW YORK

AND

UNIVERSITY OF CALIFORNIA, RIVERSIDE 



\title{
PACIFIC JOURNAL OF MATHEMATICS
}

\author{
EDITORS
}

\author{
H. SAMELSON \\ Stanford University \\ Stanford, California 94305 \\ C. R. HOBBY \\ University of Washington \\ Seattle, Washington 98105
}

J. DugundJI

Department of Mathematics

University of Southern California

Los Angeles, California 90007

RICHARD ARENS

University of California

Los Angeles, California 90024

\section{ASSOCIATE EDITORS}
E. F. BeCKenBaCH
B. H. NeUMANN
F. WOLF
K. Yoshida

\section{SUPPORTING INSTITUTIONS}

\author{
UNIVERSITY OF BRITISH COLUMBIA \\ CALIFORNIA INSTITUTE OF TECHNOLOGY \\ UNIVERSITY OF CALIFORNIA \\ MONTANA STATE UNIVERSITY \\ UNIVERSITY OF NEVADA \\ NEW MEXICO STATE UNIVERSITY \\ OREGON STATE UNIVERSITY \\ UNIVERSITY OF OREGON \\ OSAKA UNIVERSITY
}

\author{
UNIVERSITY OF SOUTHERN CALIFORNIA \\ STANFORD UNIVERSITY \\ UNIVERSITY OF TOKYO \\ UNIVERSITY OF UTAH \\ WASHINGTON STATE UNIVERSITY \\ UNIVERSITY OF WASHINGTON \\ AMERICAN MATHEMATICAL SOCIETY \\ NAVAL WEAPONS CENTER
}

The Supporting Institutions listed above contribute to the cost of publication of this Journal, but they are not owners or publishers and have no responsibility for its content or policies.

Mathematical papers intended for publication in the Pacific Journal of Mathematics should be in typed form or offset-reproduced, (not dittoed), double spaced with large margins. Underline Greek letters in red, German in green, and script in blue. The first paragraph or two must be capable of being used separately as a synopsis of the entire paper. The editorial "we" must not be used in the synopsis, and items of the bibliography should not be cited there unless absolutely necessary, in which case they must be identified by author and Journal, rather than by item number. Manuscripts, in duplicate if possible, may be sent to any one of the four editors. Please classify according to the scheme of Math. Rev. Index to Vol. 39. All other communications to the editors should be addressed to the managing editor, Richard Arens, University of California, Los Angeles, California, 90024.

50 reprints are provided free for each article; additional copies may be obtained at cost in multiples of 50 .

The Pacific Journal of Mathematics is published monthly. Effective with Volume 16 the price per volume (3 numbers) is $\$ 8.00$; single issues, $\$ 3.00$. Special price for current issues to individual faculty members of supporting institutions and to individual members of the American Mathematical Society: $\$ 4.00$ per volume; single issues $\$ 1.50$. Back numbers are available.

Subscriptions, orders for back numbers, and changes of address should be sent to Pacific Journal of Mathematics, 103 Highland Boulevard, Berkeley, California, 94708.

PUBLISHED BY PACIFIC JOURNAL OF MATHEMATICS, A NON-PROFIT CORPORATION

Printed at Kokusai Bunken Insatsusha (International Academic Printing Co., Ltd.), 270, 3chome Totsuka-cho, Shinjuku-ku, Tokyo 160, Japan. 


\section{Pacific Journal of Mathematics}

\section{Vol. 39, No. $3 \quad$ July, 1971}

William O'Bannon Alltop, 5-designs in affine spaces ................... 547

B. G. Basmaji, Real-valued characters of metacyclic groups ................. 553

Miroslav Benda, On saturated reduced products....................... 557

J. T. Borrego, Haskell Cohen and Esmond Ernest Devun, Uniquely representable semigroups. II.......................................

George Lee Cain Jr. and Mohammed Zuhair Zaki Nashed, Fixed points and stability for a sum of two operators in locally convex spaces ....................

Donald Richard Chalice, Restrictions of Banach function spaces ...............

Eugene Frank Cornelius, Jr., A generalization of separable groups ..............

Joel L. Cunningham, Primes in products of rings ......................

Robert Alan Morris, On the Brauer group of $Z$.

593

603

615

David Earl Dobbs, Amitsur cohomology of algebraic number rings ...............

Charles F. Dunkl and Donald Edward Ramirez, Fourier-Stieltjes transforms and

weakly almost periodic functionals for compact groups ...................

Hicham Fakhoury, Structures uniformes faibles sur une classe de cônes et

d'ensembles convexes ......................................

Leslie R. Fletcher, A note on C $\theta \theta$-groups.

Humphrey Sek-Ching Fong and Louis Sucheston, On the ratio ergodic theorem for

semi-groups............................................

James Arthur Gerhard, Subdirectly irreducible idempotent semigroups ...........

Thomas Eric Hall, Orthodox semigroups.....................

Marcel Herzog, $C \theta \theta$-groups involving no Suzuki groups ..........

669

687

John Walter Hinrichsen, Concerning web-like continua ..........

691

Frank Norris Huggins, A generalization of a theorem of F. Riesz.

695

Carlos Johnson, Jr., On certain poset and semilattice homomorphisms

703

Alan Leslie Lambert, Strictly cyclic operator algebras ...........

717

Howard Wilson Lambert, Planar surfaces in knot manifolds . . .

727

Robert Allen McCoy, Groups of homeomorphisms of normed linear spaces ....... 735

T. S. Nanjundiah, Refinements of Wallis's estimate and their generalizations ...... 745

Roger David Nussbaum, A geometric approach to the fixed point index .......... 751

John Emanuel de Pillis, Convexity properties of a generalized numerical range .... 767

Donald C. Ramsey, Generating monomials for finite semigroups ....

783

William T. Reid, A disconjugacy criterion for higher order linear vector differential equations...

Roger Allen Wiegand, Modules over universal regular rings...

Kung-Wei Yang, Compact functors in categories of non-archimedean Banach

spaces.

R. Grant Woods, Correction to: "Co-absolutes of remainders of Stone-Čech compactifications".

Ronald Owen Fulp, Correction to: "Tensor and torsion products of

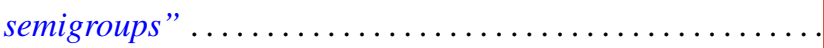

Bruce Alan Barnes, Correction to: "Banach algebras which are ideals in a banach 